\title{
Boosting Potassium Storage Performance of Red Phosphorous via Modifying Nickel Foam Substrate
}

\author{
Xian Lu ${ }^{1}$, Wei-Lin Lin ${ }^{1}$, Yue-e Huang ${ }^{1}$, Jia-Xiang Zhang ${ }^{1}$, Lun-Hui Guan², \\ Xiao-Ying Huang ${ }^{3}, \mathrm{Ke}-$ Zhao $\mathrm{Du}^{* 1}$ and Xiao-Hui $\mathrm{Wu}^{* 1}$
}

1. College of chemistry and materials science, Fujian Provincial Key Laboratory of Polymer Materials, Fujian normal university, Fuzhou, 350002, China

2. CAS key laboratory of design and assembly of functional nanostructures, Fujian key laboratory of nanomaterials, Fujian institute of research on the structure of matter, Chinese academy of sciences, Fuzhou, 350002, China

3. State key laboratory of structural chemistry, Fujian institute of research on the structure of matter, Chinese academy of sciences, Fuzhou, 350002, China

Corresponding authors who handle correspondence at all stages of refereeing and publication, also post-publication:

*Prof. Ke-Zhao Du, E-mail address: duke@,fjnu.edu.cn

*Prof. Xiao-Hui Wu, E-mail address: sherrywu@fjnu.edu.cn 

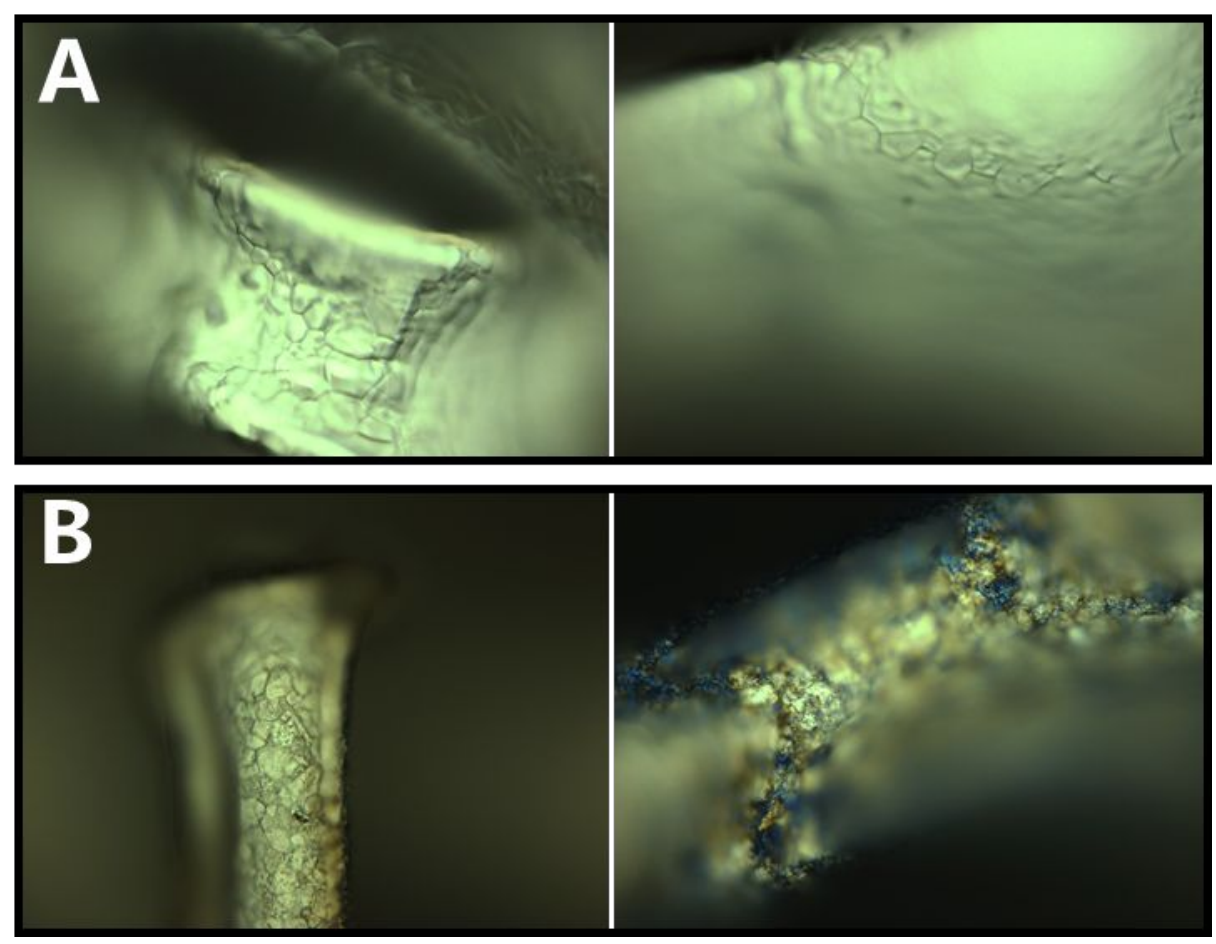

Figure S1. Metallographic microscope photographs of (A) NF and (B) h-NF.

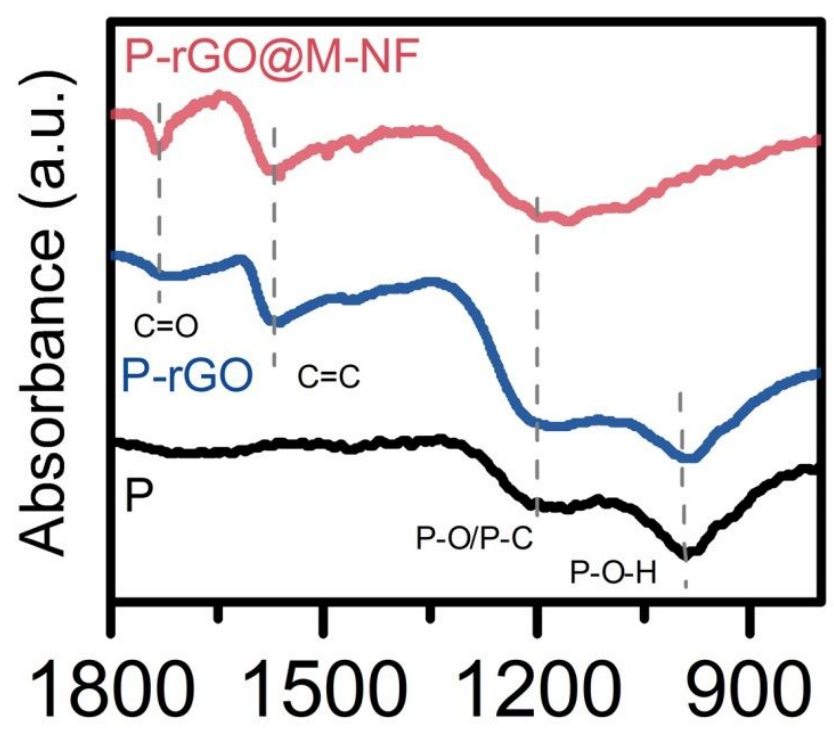

Figure S2. FT-IR spectrum of P-rGO@M-NF, P-rGO and synthesized pure RP. 


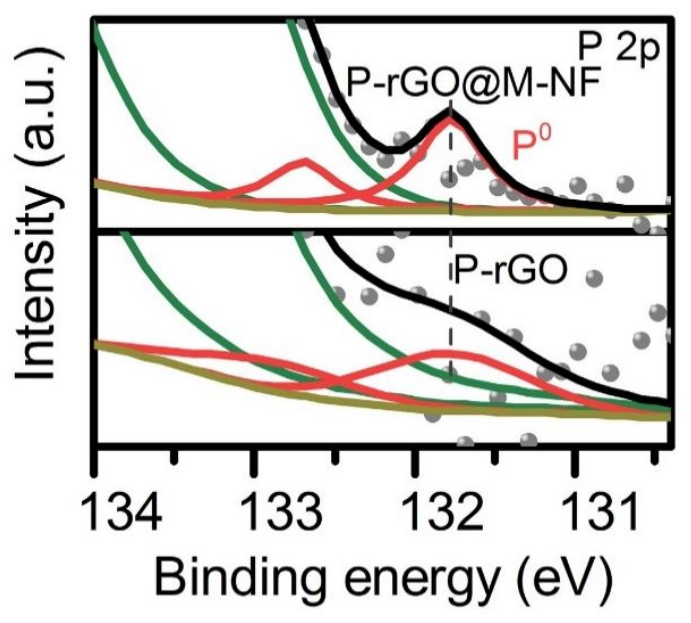

Figure S3. Magnified XPS spectra of $\mathrm{P}^{0}$ species.

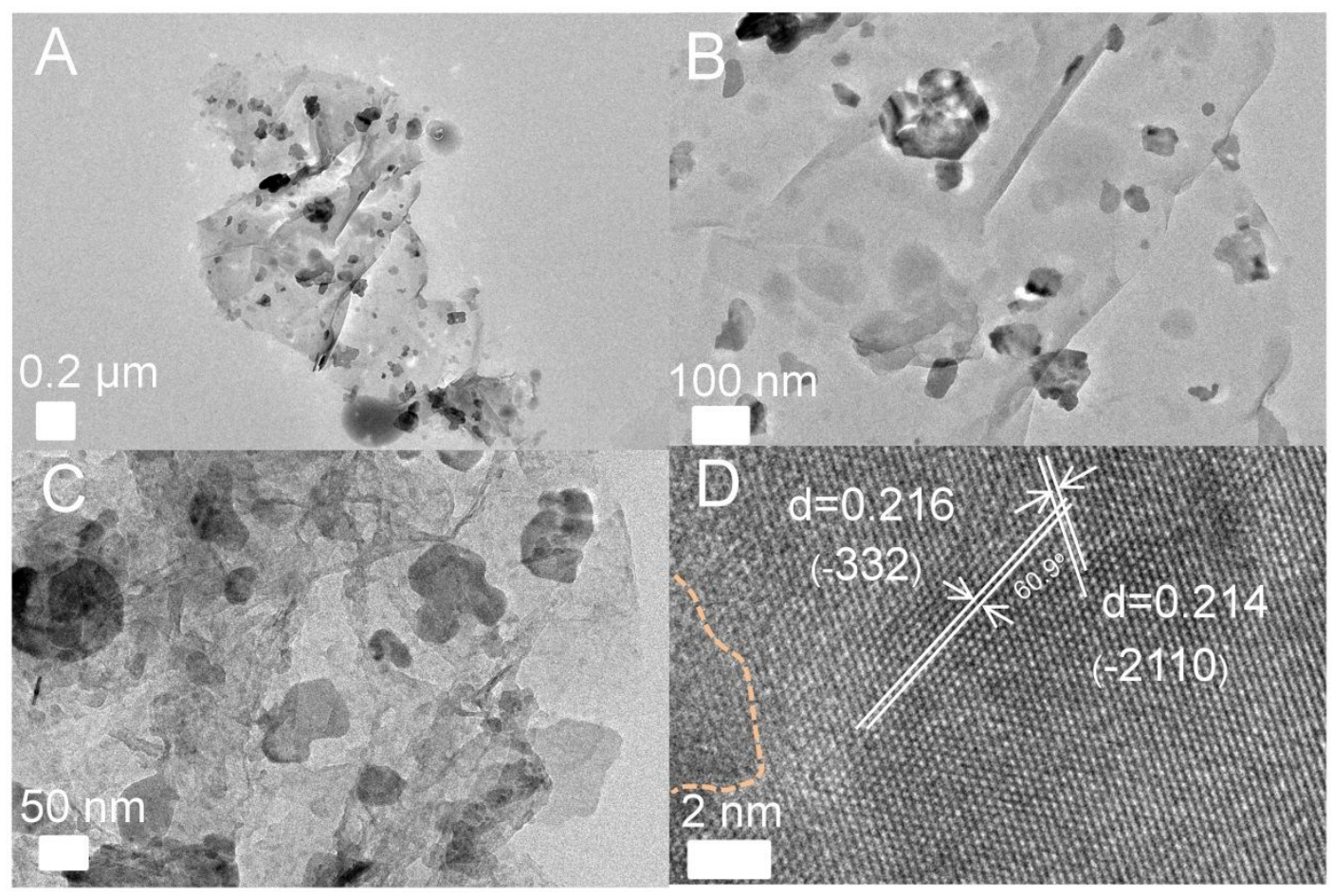

Figure S4. (A-C) TEM images and (D) high-magnification TEM image of P-rGO. 


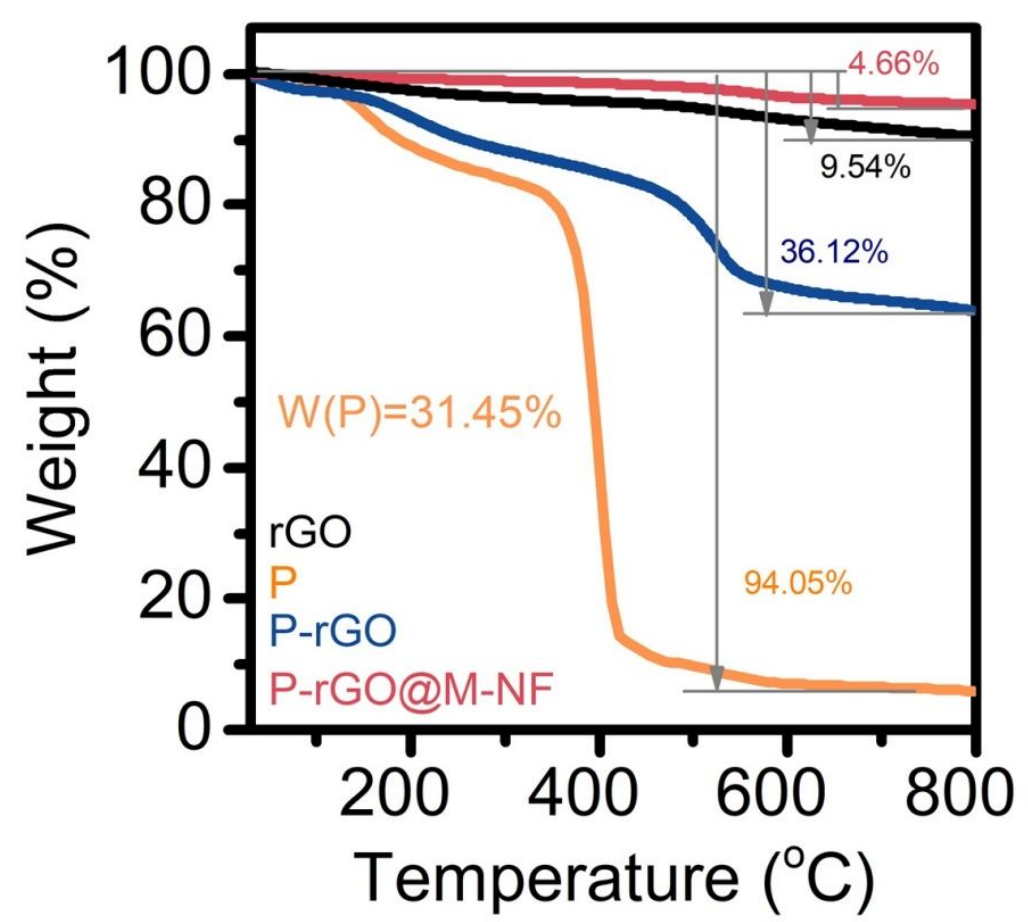

Figure S5. TGA curves of P-rGO@M-NF, P-rGO, P and rGO. The weight loss of P-rGO@M-NF, P-rGO, P and rGO are 4.66\%, 36.12\%, 94.05\% and 9.54\%.

The weight percent of $P$ in P-rGO: Assuming the content of $\mathrm{P}$ is $\mathrm{x}$ and $\mathrm{rGO}$ is (1-x) in P-rGO composite, then $94.05 \% \mathrm{x}+9.54 \%(1-\mathrm{x})=36.12 \%$. The calculated $\mathrm{x}=31.45 \%$, i.e. the weight content of $\mathrm{P}$ in $\mathrm{P}-\mathrm{rGO}$ composite is $31.45 \%$.

The weight percent of P-rGO in P-rGO@M-NF: Because the weight loss of P-rGO is $36.12 \%$ at $800^{\circ} \mathrm{C}$, the ratio of $\mathrm{P}-\mathrm{rGO}$ in should be $4.66 \% / 36.12 \%=12.90 \%$. The obtained P-rGO@M-NF is $\sim 33 \mathrm{mg}$, the weight of $\mathrm{P}-\mathrm{rGO}$ is $12.90 \% \times 33 \mathrm{mg}=4.257$ mg. The area of electrode is $1.13 \mathrm{~cm}^{2}$, so the loading of P-rGO in P-rGO@M-NF is $4.257 \mathrm{mg} / 1.13 \mathrm{~cm}^{2} \approx 3.77 \mathrm{mg} \mathrm{cm}^{-2}$. 


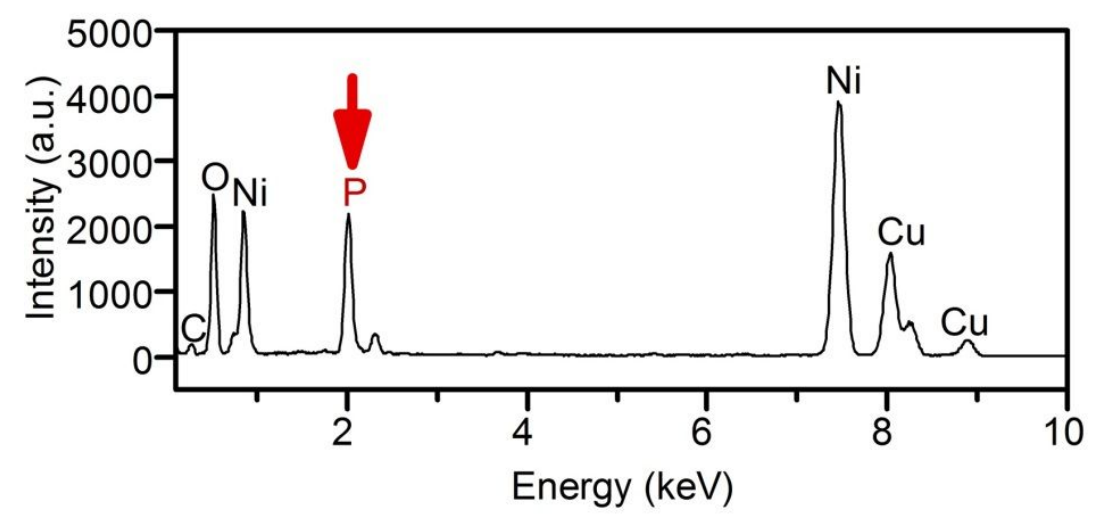

Figure S6. EDS spectra of P-rGO@M-NF.

Table S1. Element composition of P-rGO@M-NF (determined by EDS spectra).

\begin{tabular}{ccc} 
Element & Weight \% & Atomic \% \\
\hline C K & 1.59 & 4.05 \\
\hline O K & 25.89 & 49.68 \\
\hline P K & 17.79 & 17.64 \\
\hline Ni K & 54.74 & 28.63 \\
\hline
\end{tabular}




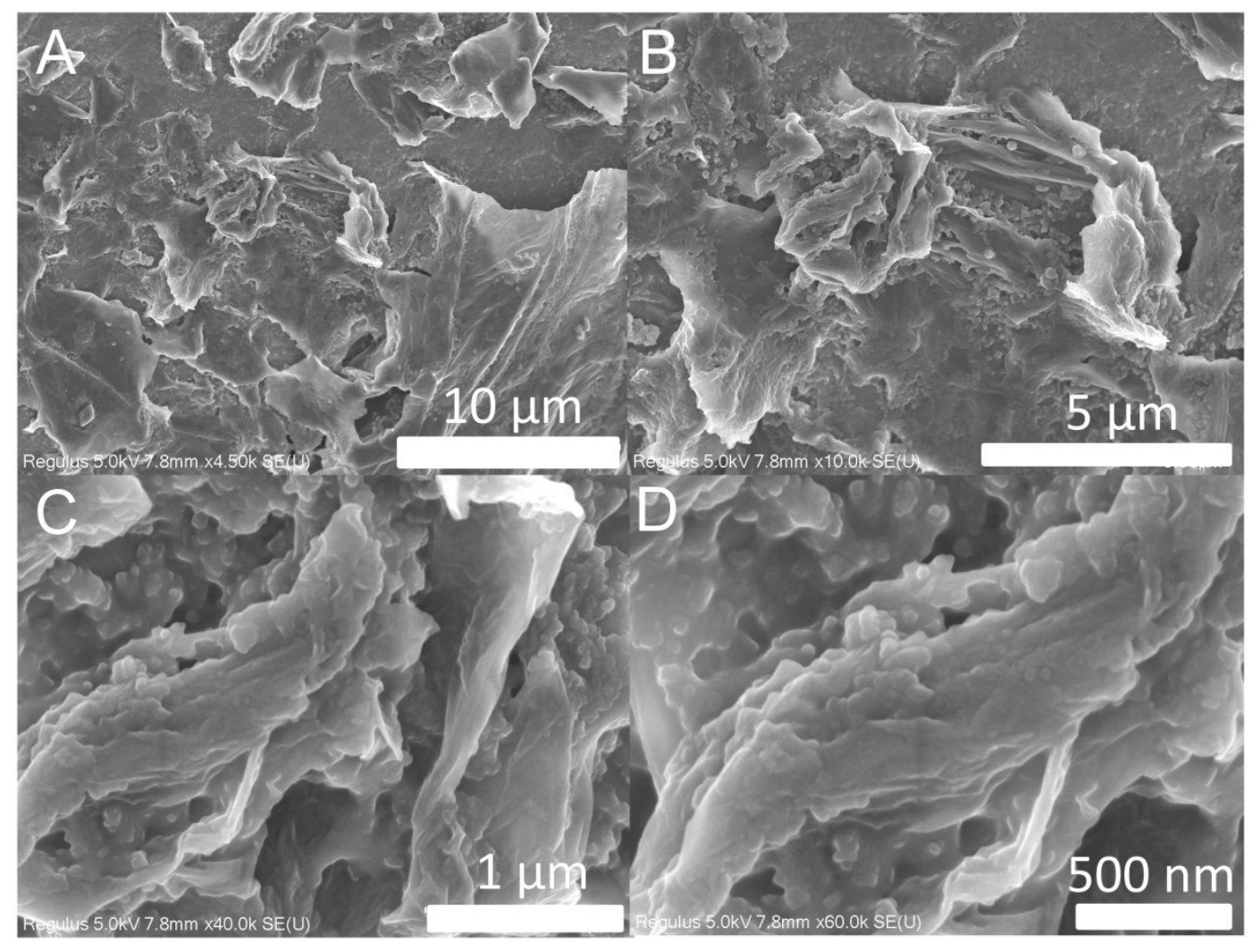

Figure S7. (A-D) SEM images of P-rGO@M-NF electrode after 900 cycles at different magnification.

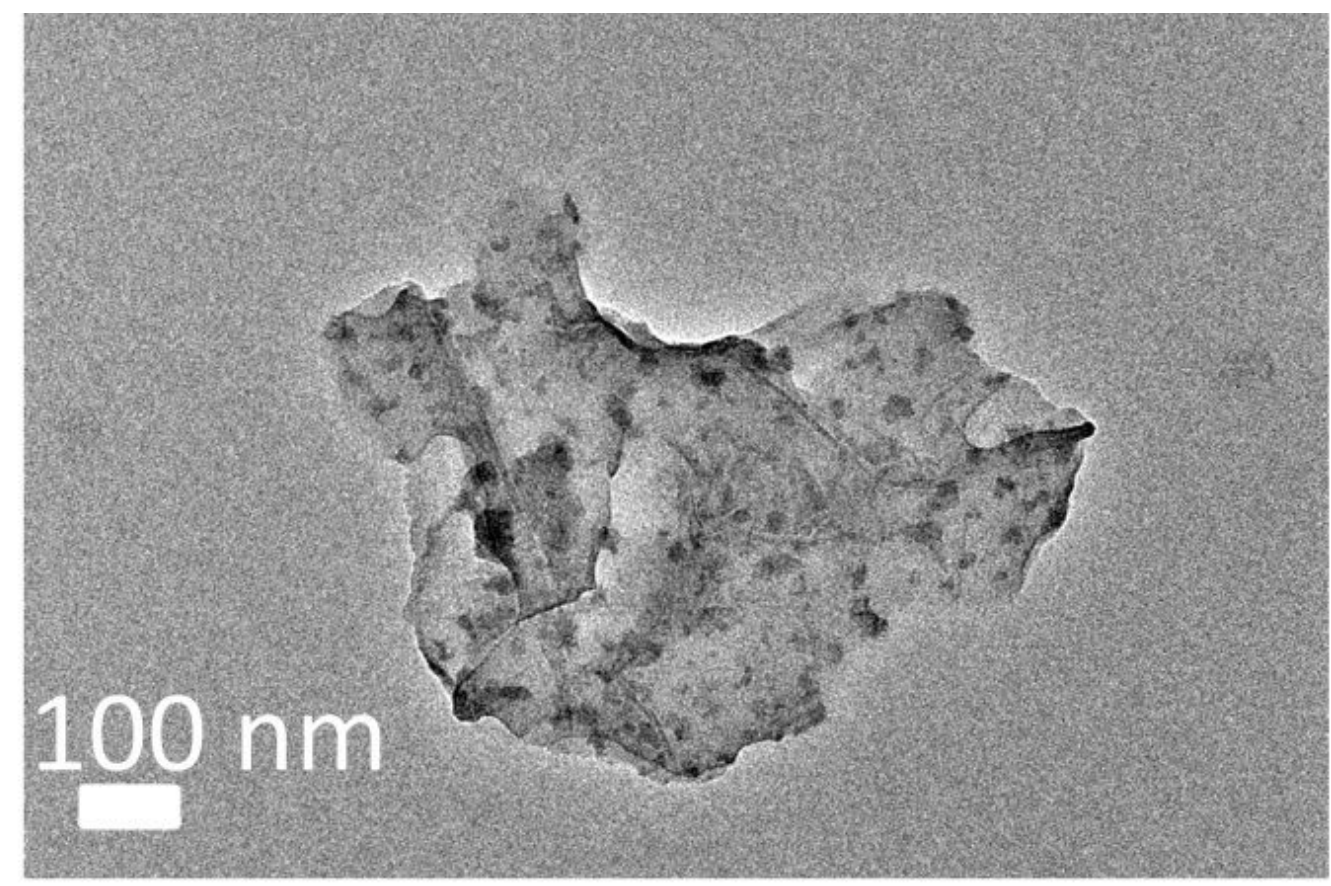


Figure S8. TEM images of P-rGO@M-NF electrode after 900 cycles.
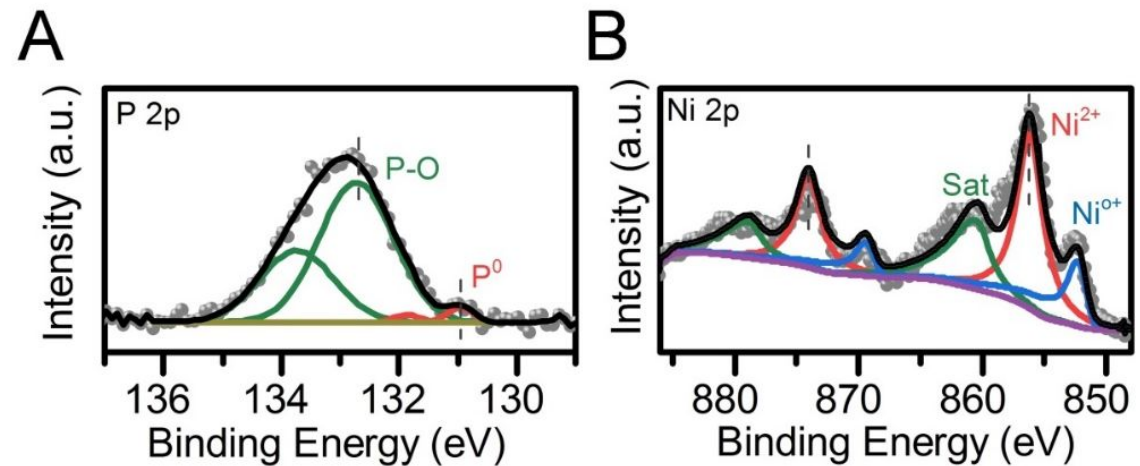

Figure S9. The high resolution XPS spectra of $\mathrm{P} 2 \mathrm{p}$ (A) and $\mathrm{Ni} 2 \mathrm{p}$ (B) for P-rGO@M-NF electrode after 900 cycles.
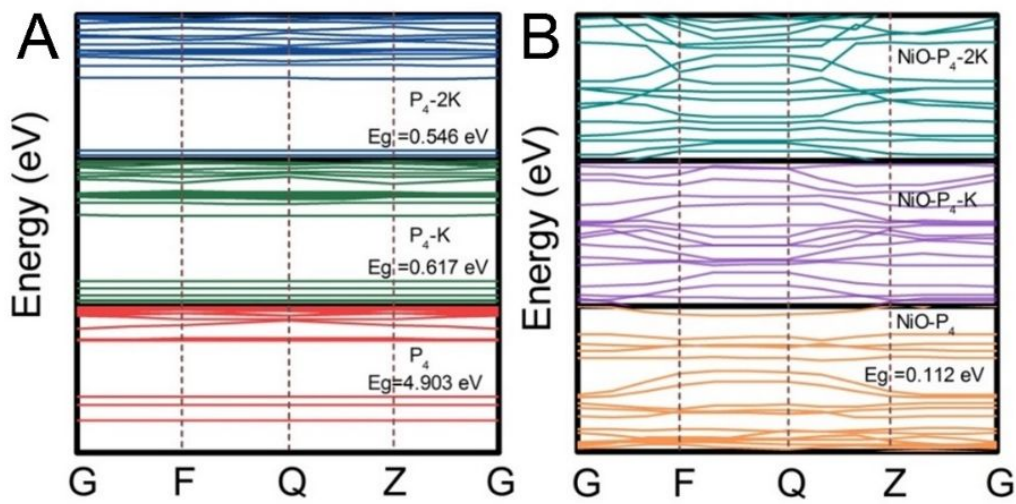

Figure S10. Band gap of (A) $\mathrm{P}_{4}, \mathrm{P}_{4}-\mathrm{K}$ and $\mathrm{P}_{4}-2 \mathrm{~K}$; (B) NiO- $\mathrm{P}_{4}, \mathrm{NiO}-\mathrm{P}_{4}-\mathrm{K}$ and NiO- $\mathrm{P}_{4}-2 \mathrm{~K}$
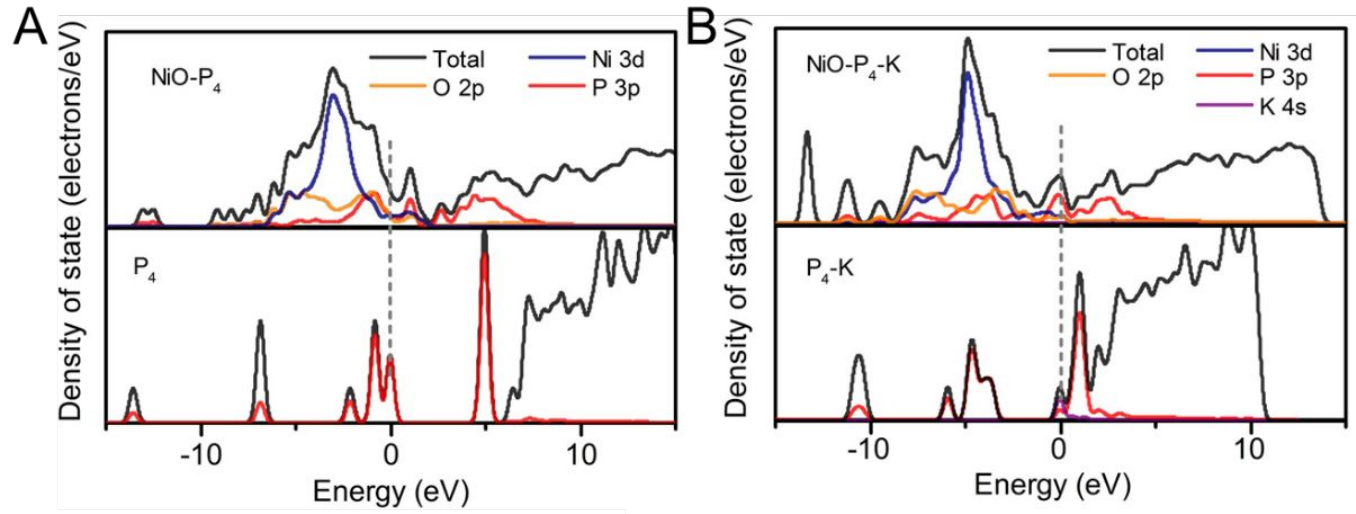
Figure S11. PDOS of (A) NiO- $\mathrm{P}_{4}$ and $\mathrm{P}_{4} ;$ (B) NiO- $\mathrm{P}_{4}-\mathrm{K}$ and $\mathrm{P}_{4}-\mathrm{K}$.

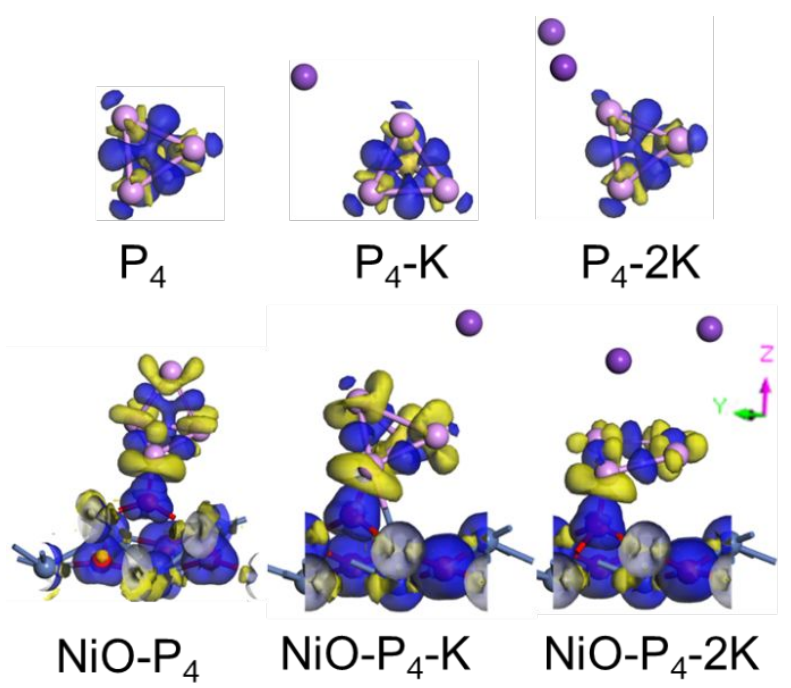

Figure S12. Differential charge densities of $\mathrm{P}_{4}, \mathrm{P}_{4}-\mathrm{K}$ and $\mathrm{P}_{4}-2 \mathrm{~K}$ (up), as well as $\mathrm{NiO}-\mathrm{P}_{4}, \mathrm{NiO}-\mathrm{P}_{4}-\mathrm{K}$ and $\mathrm{NiO}-\mathrm{P}_{4}-2 \mathrm{~K}$ (down). Blue and yellow colors indicate the electron accumulation and depletion, respectively.
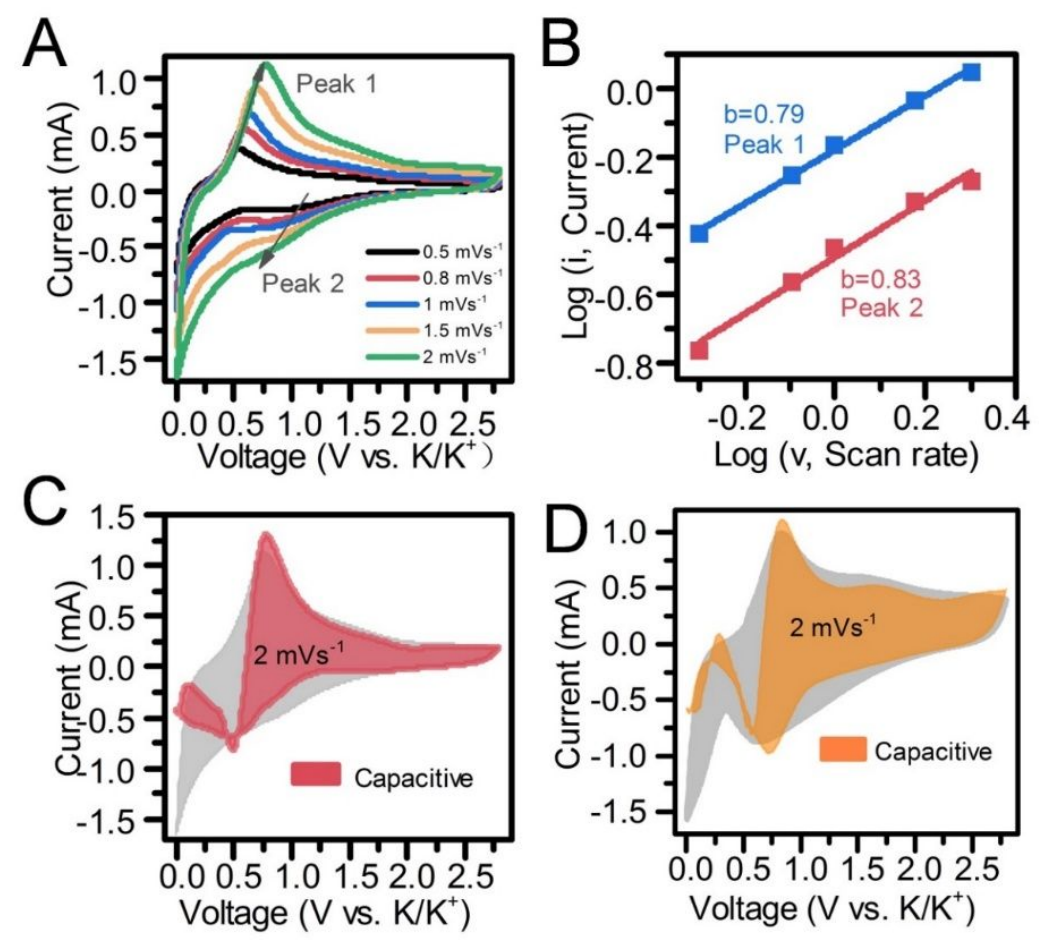

Figure S13. (A) The CV curves of P-rGO@NF at differents scan rates. (B) The calcuted $\mathrm{b}$ values of cathode peak and anode peak for P-rGO@NF electrode. The 
capacity contribution and diffusion contribution of (C) P-rGO@NF and (D) P-rGO@M-NF at a scan rate of $2.0 \mathrm{mV} \mathrm{s}^{-1}$.

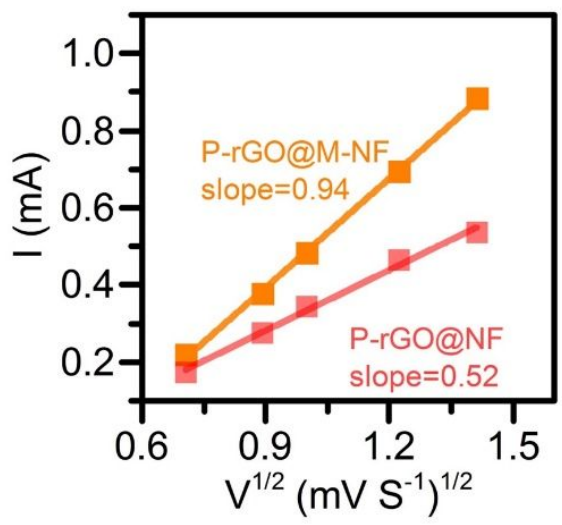

Figure S14. Corresponding relationship between square root of scanning rate and peak current for Peak 2 in P-rGO@M-NF and P-rGO@NF.

Table S2. Mulliken overlap population of P-P bond in $\mathrm{P}_{4}-2 \mathrm{~K}$ and NiO- $\mathrm{P}_{4}-2 \mathrm{~K}$.

\begin{tabular}{|c|c|c|c|}
\hline Sample & Bond & Population & Length (A) \\
\hline \multirow{6}{*}{$P_{4}-2 K$} & P2-P3 & 0.24 & 2.202 \\
\hline & P2-P4 & 0.26 & 2.204 \\
\hline & P3-P4 & 0.25 & 2.204 \\
\hline & P1-P3 & 0.18 & 2.215 \\
\hline & P1-P2 & 0.16 & 2.230 \\
\hline & P1-P4 & -0.04 & 2.309 \\
\hline \multirow{4}{*}{$\mathrm{NiO}-\mathrm{P}_{4}-2 \mathrm{~K}$} & P1-P2 & 0.5 & 2.191 \\
\hline & P1-P4 & 0.49 & 2.196 \\
\hline & P3-P4 & 0.45 & 2.196 \\
\hline & P2-P3 & 0.48 & 2.206 \\
\hline
\end{tabular}


